EPJ Web of Conferences 37, 05005 (2012)

DOI: $10.1051 /$ epjconf/20123705005

(C) Owned by the authors, published by EDP Sciences, 2012

\title{
Radiative decays of vector and pseudoscalar nonets
}

\author{
C. Terschlüsen ${ }^{1, a}$, S. Leupold ${ }^{1}$, and M.F.M. Lutz ${ }^{2}$
}

1 Institutionen för fysik och astronomi, Uppsala Universitet, Box 516, 75120 Uppsala, Sweden

2 GSI Helmholtzzentrum für Schwerionenforschung GmbH, Planckstraße 1, 64291 Darmstadt, Germany

\begin{abstract}
Using a counting scheme which treats the light pseudoscalar and the light vector-meson nonet on equal footing, the decays of the narrow light vector mesons into a pseudoscalar meson and a dilepton and of the $\eta^{\prime}$ meson into an $\omega$ meson and a dielectron are calculated. Since all required parameters but one can be determined by other reactions, one has predictive power for the considered decay rates and form factors. The calculated partial decay widths are in good agreement with the available experimental data.
\end{abstract}

\section{Introduction}

According to the vector-meson dominance conjecture [1], electromagnetic reactions of hadrons can be described efficiently with dynamical vector meson degrees of freedoms. The standard vector-mesondominance model (VMD) is able to describe many of such reactions quite successfully, but it cannot generate a consistent picture. An example of the failure of the VMD is the $\omega-\pi^{0}$ transition form factor, i.e. the transition form factor for the decay of an $\omega$ meson into a neutral pion and a dilepton [2]. In the following, we will study such transition form factors within an effective chiral Lagrangian with explicit vector meson fields [3].

As a result of the running coupling constant in QCD it is not possible to use perturbation theory for low energy hadron physics. A possible solution to this problem are effective field theories which use hadrons instead of quarks and gluons as relevant degrees of freedom. In the lowest energy range, such an effective theory is chiral perturbation theory (ChPT) $[4,5]$ which uses only the Goldstone bosons $\pi, K$ and $\eta$ as active degrees of freedom. In turn, ChPT is not applicable for the energy range of the hadronic resonances $\rho, \omega, K^{*}, \phi$ and $\eta^{\prime}$. Though it is straight forward to include the vector meson field into the chiral Lagrangian, it is an open and controversial issue how to arrive at a controlled approximation scheme given such a Lagrangian. Here we apply a novel counting scheme as proposed recently in $[6,3]$. The masses of both pseudoscalar and vector mesons, $m_{P}$ and $m_{V}$, respectively, are regarded as light implying the universal counting rules

$$
m_{P}, m_{V}, D_{\mu} \sim Q
$$

with the covariant derivative $D_{\mu}$ and any momentum $Q$. The restriction to light pseudoscalar and vector mesons as relevant degrees of freedom can by motivated within the hadrogenesis conjecture which suggests that all other low-lying mesons are dynamically generated from interactions of pseudoscalar and vector mesons $[6-9,3]$.

\footnotetext{
a e-mail: carla.terschluesen@physics.uu.se
} 


\section{Leading-order Lagrangian}

Applying the counting scheme (1), one can determine the leading-order Lagrangian for the decays $V \rightarrow P \gamma^{(*)}$ and $P \rightarrow V \gamma^{(*)}$,

$$
\begin{aligned}
\mathcal{L}= & -\frac{h_{A}}{16 f} \varepsilon^{\mu \nu \alpha \beta} \operatorname{tr}\left\{\left[\Phi_{\mu \nu}, \partial^{\tau} \Phi_{\tau \alpha}\right]_{+} \partial_{\beta} \Phi\right\}-\frac{b_{A}}{8 f} \varepsilon^{\mu \nu \alpha \beta} \operatorname{tr}\left\{\Phi_{\mu \nu}\left[\Phi, \chi_{0}\right]_{+} \Phi_{\alpha \beta}\right\} \\
& -\frac{m_{V}^{2} h_{H}}{4 f_{H}} \varepsilon^{\mu \nu \alpha \beta} \operatorname{tr}\left\{\Phi_{\mu \nu} \Phi_{\alpha \beta}\right\} \tilde{\eta}_{1}-e f_{V} \operatorname{tr}\left\{\Phi^{\mu \nu} Q\right\} \partial_{\mu} A_{v}+\frac{e f_{V} e_{H}}{f_{H}} \varepsilon^{\mu \nu \alpha \beta} \operatorname{tr}\left\{\Phi_{\mu \nu} Q\right\} \partial_{\alpha} A_{\beta} \tilde{\eta}_{1} .
\end{aligned}
$$

Hereby, the matrix $\Phi_{\mu \nu}$ describes the vector-meson nonet represented by antisymmetric tensor fields and $\Phi$ the pseudoscalar nonet. Furthermore, $\chi_{0}=\operatorname{diag}\left(\mathrm{m}_{\pi}^{2}, \mathrm{~m}_{\pi}^{2}, 2 \mathrm{~m}_{\mathrm{K}}^{2}-\mathrm{m}_{\pi}^{2}\right)$ denotes the mass matrix, $Q=\operatorname{diag}(2 / 3,-1 / 3,-1 / 3)$ the quark-charge matrix and $A_{\mu}$ the photon field.

Just decays including the $\eta$ singlet state, $\tilde{\eta}_{1}$, can happen directly. The corresponding vertices are given by the terms which include the $\tilde{\eta}_{1}$ explicitly (proportional to $h_{H}$ and $e_{H}$ ). Otherwise, this Lagrangian allows only indirect decays, i.e. decays via a virtual vector meson. The decay of the virtual photon into a dilepton is described by usual QED.

The pseudoscalar nonet $\Phi$ in the Lagrangian (2) includes the non-physical singlet state $\tilde{\eta}_{1}$ and octet state $\eta_{8}$

$$
\Phi=\eta_{8} \lambda^{8}+\tilde{\eta}_{1} \frac{f}{f_{H}} \lambda^{0}
$$

Therewith, the physical fields $\eta$ and $\eta^{\prime}$ are defined as linear combination of the non-physical states,

$$
\eta=-\tilde{\eta}_{1} \sin \theta+\eta_{8} \cos \theta, \quad \eta^{\prime}=\tilde{\eta}_{1} \cos \theta+\eta_{8} \sin \theta,
$$

with a to be determined mixing angle $\theta$. Since the physical fields are defined as non-mixing, the (leading-order) mixing angle can be calculated from the $\eta$ and $\eta^{\prime}$ masses using the leading-order free Lagrangian for the pseudoscalar nonet. One gets $\theta \approx-11^{\circ}$. However, if the squared sum of the $\eta$ and $\eta^{\prime}$ masses is kept at its empirical value the $\eta$ and $\eta^{\prime}$ masses can be calculated as functions of the mixing angle. For $|\theta| \leq 15^{\circ}$, the discrepancy between theoretical and experimental masses is less than $5 \%$. Hence, we use the mixing angle as an additional open parameter.

If one uses the $\eta-\eta^{\prime}$ mixing angle $\theta$ as an additional parameter, the leading-order Lagrangian (2) has six open parameters. All parameters except $f_{H}$ are determined from five two-body decays,

$$
\omega \rightarrow \pi^{0} \gamma, \quad \omega \rightarrow \eta \gamma, \quad \phi \rightarrow \eta \gamma, \quad \phi \rightarrow \eta^{\prime} \gamma, \eta^{\prime} \rightarrow \omega \gamma,
$$

by comparing the calculated squared matrix element with experimental data. Since only absolute values for the matrix elements can be compared, one gets four parameter sets if one restricts the parameter $h_{A}$ to be positive. These four sets can be reduced to two sets with "reasonable" parameters compared to previous calculations without $\eta^{\prime}[6,10,11]$,

$$
\begin{array}{ll}
\theta= \pm 2^{\circ}, \quad h_{A}=2.33, & b_{A}=0.16, \\
h_{H}=0.14 \mp 0.19 \frac{f_{H}}{f}, & e_{H}=-0.20 \mp 0.70 \frac{f_{H}}{f} .
\end{array}
$$

In the following, the undetermined parameter $f_{H}$ will be varied between the pion decay constant $f$ and $\sqrt{2} f^{1}$. The two parameter sets can be distinguished by the sign of the mixing angle. Thereby, only the parameters $h_{H}$ and $e_{H}$ from the terms with an explicit $\eta$ singlet differ for the two parameter sets and depend on the undetermined parameter $f_{H}$ whereas the parameters $h_{A}$ and $b_{A}$ remain unchanged. For the decays into dileptons no additional parameters are needed so that we have real predictive power for such decays.

\footnotetext{
1 Note that the vector-meson decay constant $f_{V} \approx \sqrt{2} f$.
} 

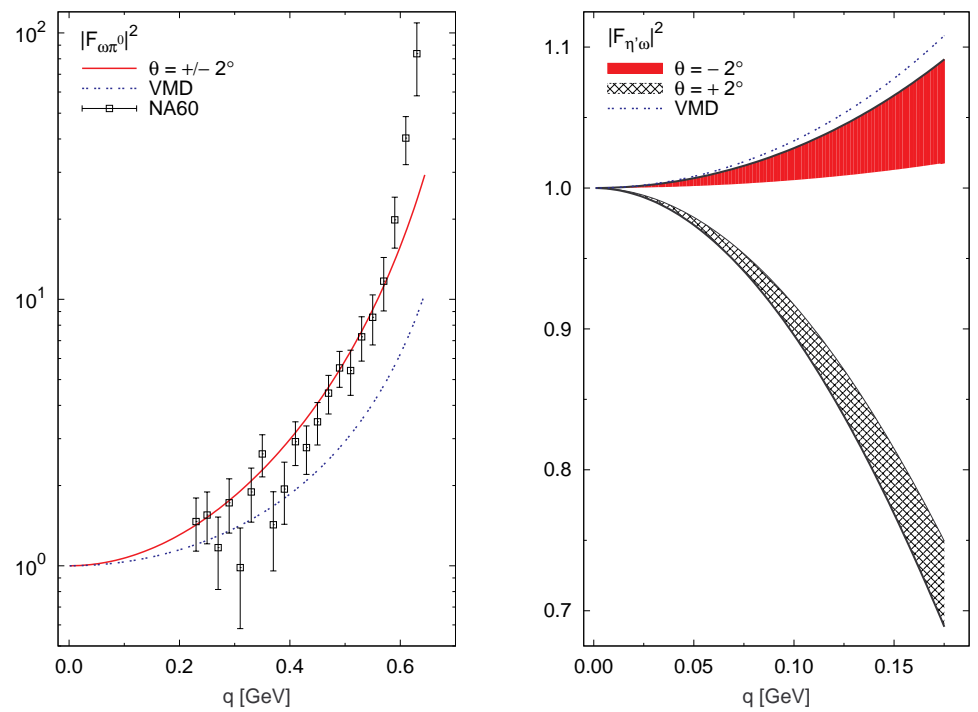

Fig. 1. Left: Form factor for the decay $\omega \rightarrow \pi^{0} l^{+} l^{-}$compared to the dimuon data taken by the NA60 collaboration [2]. Right: Form factor for the decay $\eta^{\prime} \rightarrow \omega e^{+} e^{-}$. The solid lines describe the results for $f_{H}=f$.

\section{Results}

Due to isospin conservation, the transition $\omega \rightarrow \pi^{0}$ is only possible via a virtual $\rho^{0}$ meson. Thus, VMD predicts the form factor

$$
F_{\omega \pi^{0}}^{\mathrm{VMD}}(q)=\frac{m_{\rho}^{2}}{m_{\rho}^{2}-q^{2}}
$$

depending on the invariant dilepton mass $\sqrt{q^{2}}$. Our calculation yields an additional constant term,

$$
F_{\omega \pi^{0}}(q) \sim h_{A}+\frac{-\left(m_{\omega}^{2}+m_{\rho}^{2}\right) h_{A}+8 b_{A} m_{\pi}^{2}}{m_{\rho}^{2}-q^{2}} .
$$

The proportionality factor is fixed by the normalisation condition $F_{\omega \pi^{0}}(0)=1$.

On the left-hand side in fig. 1, this form factor is plotted in comparison to the data taken by the NA60 collaboration and the VMD form factor (7). As already mentioned in the introduction, VMD fails to describe the data whereas our calculation misses only the last three data points. The reduced $\chi^{2} / N$ for the single-differential decay width for our approach, $\chi^{2} / N=1.8$, is much smaller than the one for $\mathrm{VMD}, \chi_{\mathrm{VMD}}^{2} / N=4.8$. Furthermore, the partial widths for the decays into dimuon and dielectron agree very well with the experimental data,

$$
\begin{aligned}
& \Gamma_{\omega \rightarrow \pi^{0} \mu^{+} \mu^{-}}=(9.74 \pm 0.30) \cdot 10^{-7} \mathrm{GeV}, \\
& \Gamma_{\omega \rightarrow \pi^{0} \mu^{+} \mu^{-}}^{\exp }=(11.04 \pm 3.40) \cdot 10^{-7} \mathrm{GeV}, \\
& \Gamma_{\omega \rightarrow \pi^{0} e^{+} e^{-}}=(6.85 \pm 0.21) \cdot 10^{-6} \mathrm{GeV}, \\
& \Gamma_{\omega \rightarrow \pi^{0} e^{+} e^{-}}^{\exp }=(6.54 \pm 0.51) \cdot 10^{-6} \mathrm{GeV} .
\end{aligned}
$$

For the decay of an $\eta^{\prime}$ meson, $\eta-\eta^{\prime}$ mixing has to be taken into account. Thus, the form factor is given by

$$
F_{\eta^{\prime} \omega} \sim \sin \theta \hat{f}_{\eta_{8} \omega}+\frac{f}{f_{H}} \cos \theta \hat{f}_{\eta_{1} \omega}
$$


including the form factors for the formal decays of the octet and singlet states,

$$
\begin{aligned}
& \hat{f}_{\eta_{8} \omega}(q)=h_{A}+\frac{-2 m_{\omega}^{2} h_{A}+8 m_{\pi}^{2} b_{A}}{m_{\omega}^{2}-q^{2}}, \\
& \hat{f}_{\eta_{8} \omega}(q)=\sqrt{2}\left\{h_{A}-2 \sqrt{6} e_{H}+\frac{-2 m_{\omega}^{2} h_{A}+8 m_{\pi}^{2} b_{A}+4 \sqrt{6} m_{V}^{2} h_{H}}{m_{\omega}^{2}-q^{2}}\right\} .
\end{aligned}
$$

The form factor is plotted on the right-hand side in fig. 1 in comparison to the VMD calculation. Here, the two parameter sets $\theta= \pm 2^{\circ}$ are clearly distinguishable and an uncertainty caused by $f_{H}$ can be observed. However, for both parameter sets there is a (clear) deviation from VMD visible.

Data for the $\eta^{\prime}-\omega$ transition form factor could be used to determine the sign of the mixing angle, i.e. single out the parameter set, and to determine the open parameter $f_{H}$. Unfortunately, data for the decay $\eta^{\prime} \rightarrow \omega e^{+} e^{-}$is not available at present. Yet, we predict a relatively large branching ratio,

$$
\mathrm{Br}_{\eta^{\prime} \rightarrow \omega \mathrm{e}^{+} \mathrm{e}^{-}}=(1.69 \pm 0.56) \cdot 10^{-4}
$$

\section{Summary}

We discussed the implications of a new counting scheme which treats the nonets of both the light pseudoscalar and vector mesons on the same footing. The partial decay width for the decay $\omega$ into neutral pion and dimuon or dielectron in leading order are found in good agreement with the experimental data. In particular, the $\omega-\pi^{0}$ transition form factor is much better described in our scheme than within the standard VMD model. Furthermore, we predicted the leading-order branching ratio for the decay $\eta^{\prime} \rightarrow \omega e^{+} e^{-}$. The decays presented here are just examples, in addition the decays $\omega \rightarrow \eta l^{+} l^{-}$, $\phi \rightarrow \eta l^{+} l^{-}$and $\phi \rightarrow \eta^{\prime} e^{+} e^{-}$have been calculated in [3].

\section{References}

1. J.J. Sakurai, Currents and mesons (University of Chicago Press, Chicago, USA, 1969).

2. R. Arnaldi et al., Phys. Lett. B 277, (2009) 260.

3. C. Terschlüsen, S. Leupold, M.F.M. Lutz, arXiv:1204.4125 [hep:ph]

4. J. Gasser and H. Leutwyler, Nucl. Phys. B 250, (1985) 465.

5. S. Scherer, Adv. Nucl. Phys. 27, (2003) 277.

6. M.F.M. Lutz and S. Leupold, Nucl. Phys. A 813, (2008) 96.

7. M.F.M. Lutz and E. E. Kolomeitsev, Nucl. Phys. A 730 (2004) 392.

8. M.F.M. Lutz and M. Soyeur, Nucl. Phys. A813, (2008) 14.

9. I.V. Danilkin, L.I.R. Gil and M.F.M. Lutz, Phys. Lett. B703 (2011)

10. S. Leupold and M.F.M. Lutz, Eur. Phys. A 39, (2009) 205

11. C. Terschlüsen and S. Leupold, Phys. Lett. B 691, (2010) 191 